\title{
Improved Grid Optimization and Fitting in Least Squares Tensor Hypercontraction
}

\author{
Devin A. Matthews* \\ Southern Methodist University, Dallas, TX 75275, USA \\ E-mail: damatthews@smu.edu
}

\begin{abstract}
A new method for generating fitting grids for least-squares tensor hypercontraction (LS-THC) is presented. This method draws inspiration from the related interpolative separable density fitting (ISDF) technique, but uses only a pivoted Cholesky decomposition of the metric matrix, $S$, already computed as a matter of course in LS-THC. The size and quality of the resulting grid is controlled by a user-defined cutoff parameter and the size of the starting grid. Additionally, the Cholesky-based method provides an alternative and possible more numerically stable method for performing the least-squares fit. The quality of the grids produced is evaluated for LS-DF-THC-MP2 calculations on retinal and benzene, the former with a large starting grid and small cc-pVDZ basis set, and the latter with a wide range of grids and basis sets. The error and grid size is found to be well-controlled by either the cutoff parameter (with a large starting grid) or the starting grid size (with a tight cutoff) and highly predictable. The Cholesky-based method is also able to generate unique grids tailored to different charge distributions, for example the $(a b \mid,(a i)$, and $(i j \mid$ distributions that arise in the molecular orbital integrals. While only the $(a i \mid$ grid directly affects the MP2 energy, the relative sizes of the other grids are examined.
\end{abstract}




\section{Introduction}

Tensor hypercontraction (THC) $\underline{1}$ is a promising tensor factorization technique that is, in theory, applicable to any wavefunction-based electronic structure method. The original conception of THC approximately factorized the two-electron atomic orbital (AO) integrals into a product of five matrices,

$$
(\mu \nu \mid \rho \sigma) \approx \sum_{P Q} X_{\mu}^{P} X_{\nu}^{P} V_{P Q} X_{\rho}^{Q} X_{\sigma}^{Q}
$$

The structure of this approximation is similar in structure to a double pseudo-spectral decomposition,,$^{2-6}$ although the $\operatorname{method}(\mathrm{s})$ for determining the values of the collocation matrices $X$ and core matrix $V$ differ from those used in pseudo-spectral theory as do their numerical behavior. Even more closely related is the semi-numeric chain-of-spheres exchange (COSX)

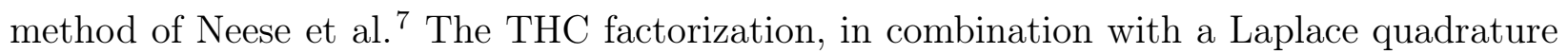
of the orbital energy denominators was used to derive reduced-scaling approaches to MP2, $\underline{1}, \underline{\underline{8}}$ MP3, ${ }^{1}$ CC2,,$\frac{9,10}{2}$ and the expensive particle-particle ladder term in CCSD. $\stackrel{11}{ }$ Recent work on the application of this factorization scheme to the coupled cluster doubles amplitudes, $\hat{T}_{2}, \underline{12}$ as well as the two-electron integrals has also lead to reduced scaling CC approaches such as Tensor-Structured CC 13 as well as THC versions of CASPT2, $\stackrel{14}{\underline{p} \text { pRPA, }} \stackrel{15}{\underline{1}}$ and p2RDM methods. $\stackrel{16}{ }$ Parrish et al. also showed that accuracy could be improved by directly fitting the molecular orbital (MO) integrals $(p q \mid r s)$ rather than transforming the factorization of the AO integrals after the fact. $\underline{8,11}$

In the least-squares variant of THC (LS-THC), $\underline{\underline{8}}$ a global solution to (1) via non-linear optimization is abandoned in favor of an ansatz in which the matrix $X$ is fixed by the choice of a grid $\left\{x_{P}\right\}_{P=1}^{n_{P}}$ and weights $\omega_{P}$ such that $X_{\mu}^{P}=\omega_{P} \phi_{\mu}\left(x_{P}\right)$. However, this approach is complicated by two factors: first, the choice of the molecular grid is critical - it must be large enough to accurately represent the electron-electron interaction, but not so large that the cost of the calculation balloons or numerical issues are encountered. $\underline{17}, \underline{18}$ Second, the LS-THC 
procedure requires the inversion of a "metric matrix", $S$, which must be handled with care. This matrix is generally rank-deficient (and hence singular), such that a pseudoinverse must be constructed rather than a conventional inverse.

Lu and Ying worked around these problems, as well as the $\mathscr{O}\left(n^{4}\right)$ or $\mathscr{O}\left(n^{5}\right)$ cost of building the $E$ matrix, in the context of periodic calculations. $\frac{19}{}$ They developed a method which they term the Interpolative Separable Density Fitting (ISDF), where a randomized QR factorization of the joint collocation matrix $Y_{\mu \nu}^{P}=X_{\mu}^{P} X_{\nu}^{P}$ is used to build a set of auxiliary

functions $\tilde{\phi}_{P}$ that define the Coulomb kernel $V_{P Q}=\left(\tilde{\phi}_{P} \mid \tilde{\phi}_{Q}\right)$. This approach proved fruitful for reducing the cost of calculating Hartree-Fock exchange $\frac{19}{9}$ and $\mathrm{RPA}^{20}$ correlation energies, but the application of ISDF to molecular systems is far less straightforward due to the difficulty of computing the singular integrals required for the Coulomb kernel.

In this Letter, we present a technique that bridges the "classic" LS-THC method with ideas from ISDF to arrive at a technique that is capable of automatically determining optimized (pruned) grids specific to a given $(p q \mid$ molecular orbital charge distribution. The quality and size of the generated grids are investigated as a function of a user-defined threshold $\epsilon$ and the parent grid size. This technique is shown to produce high-quality grids with a much smaller number of grid points than in the original grid, and to scale effectively to basis sets as large as cc-pV6Z. The procedure for determining the pruned grids also leads to an alternate method for performing the least-squares fit which may be more numerically stable.

\section{Theory}

The LS-THC procedure is characterized by a closed-form solution to the THC fit, $\underline{8}$

$$
\begin{aligned}
V & =S^{-1} E S^{-1} \\
S_{P^{\prime} Q^{\prime}} & =\sum_{p q} X_{p}^{P^{\prime}} X_{q}^{P^{\prime}} X_{p}^{Q^{\prime}} X_{q}^{Q^{\prime}}
\end{aligned}
$$


where $X$ is the (parent) grid collocation matrix, here in the MO basis. The fitting matrix $E$ may be determined in a number of ways; the density fitting approximation $\underline{21} \underline{22}$ is used in this work.

In the ISDF approach, $\underline{\underline{19}}$ the density is fit by an implicit auxiliary basis $\tilde{\phi}_{P}\left(x_{P^{\prime}}\right)$ defined only at a set of grid points. The auxiliary functions are determined by a randomized sampled QR procedure,

$$
M=\mathcal{S P} Y, \quad M \Pi=Q R
$$

where the joint collocation matrix $Y$ in the starting grid is stored as $Y_{p q, P}$. $\mathcal{P}$ is a permutation or mixing matrix (e.g. FFT). $\mathcal{S}$ is a selection matrix which retains a random set of $r N$ rows, where $r$ is an oversampling parameter and $N$ is the number of MOs. Finally, $M \Pi=$ $Q R$ computes the $\mathrm{QR}$ decomposition with column pivoting. The auxiliary basis functions are then formed by selecting $n_{P} \leq n_{P^{\prime}}$ such that $\left|R_{n_{P}+1, n_{P}+1}\right|<\epsilon\left|R_{1,1}\right| \leq\left|R_{n_{P}, n_{P}}\right|$ and computing,

$$
\tilde{X}=R_{1: n_{P}, 1: n_{P}}^{-1} R_{1: n_{P},:} \Pi^{-1}
$$

where $\tilde{X}_{P P^{\prime}}=\tilde{\phi}_{P}\left(x_{P^{\prime}}\right)$. Together with explicit Fourier-space integration of $V_{P Q}=\left(\tilde{\phi}_{P} \mid \tilde{\phi}_{Q}\right)$ via $\tilde{X}$ this gives a THC-like decomposition.

Rather than apply ISDF directly to molecules, it is instead interesting to draw further parallels between ISDF and LS-THC by examining the $M$ matrix. In fact, there is a close link between this quantity and the metric matrix, $S$,

$$
\begin{aligned}
M^{T} M & =Y^{T} \mathcal{P}^{T} \mathcal{S}^{T} \mathcal{S P Y} \\
& \approx Y^{T} Y=S
\end{aligned}
$$


Additionally, the QR decomposition of $M$ may also be converted to a decomposition of $S$,

$$
\begin{aligned}
S \approx M^{T} M & =\Pi R^{T} Q^{T} Q R \Pi^{-1} \\
& =\Pi R^{T} R \Pi^{-1}
\end{aligned}
$$

Thus, the $R$ factor can equivalently be obtained by a pivoted Cholesky decomposition. $\underline{\underline{23}}$ As in ISDF, only the leading portion of $R$ (using the same cutoff criterion and threshold $\epsilon$ ) is numerically relevant to the computation. The selected rows of $R$ also define the pruned grid $\left\{x_{P}\right\}$ from the original grid $\left\{x_{P^{\prime}}\right\}$ and the pruned collocation matrix $X_{p}^{P}$ from $X_{p}^{P^{\prime}}$. Using different combinations of $X_{a}^{P^{\prime}}$ and $X_{i}^{P^{\prime}}$ we can build three distinct $S$ matrices corresponding to the $(a b \mid,(a i \mid$, and $(i j \mid$ distributions. Each of these results in a unique pruned grid.

The Cholesky decomposition procedure allows for several critical optimizations. Because the Cholesky factorization proceeds incrementally, we can compute successive rows of $R$ until the diagonal falls below the threshold and then stop early. In contrast, one must generally compute all of the eigenvalues during pseudoinversion. Similarly, while both algorithms scale as $\mathscr{O}\left(n^{3}\right)$ the Cholesky factorization has a much lower constant factor than eigendecomposition. However, the main benefit of this approach compared to pseudoinversion is that pruning the grid leads to a reduction in the cost of all following computations (building the $E$ matrix, fitting, and the THC computation). The Cholesky decomposition is a necessary factor for this optimization, as the leading eigenvectors of $S$ may be arbitrary linear combinations of grid points. These non-local functions are then no longer suitable for defining an auxiliary basis since they destroy the property $(\mu \nu P)=(\mu P)(\nu P)=X_{\mu}^{P} X_{\nu}^{P}$. In addition to decreasing computational cost, the use of the Cholesky factorization may also improve numerical stability in the least squares fitting solution. Instead of explicitly computing $S^{-1}=R^{-1} R^{-T}$ in the solution of $V=S^{-1} E S^{-1}$, we may instead solve the system of equations $S V S=R^{T} R V R^{T} R=E$ using four triangular solves and successive back-substitution. For ill-conditioned matrices, forward solves are generally preferable to 
explicit inversion where possible. Full pseudocode for the Cholesky-based LS-THC fitting procedure is given in the Supporting Information.

\section{Results}

Since the Cholesky procedure can select an optimal (in some sense) sub-grid from the parent grid, a natural question to ask is, "Given a large enough starting grid, how does the pruned grid size and accuracy depend on the cutoff parameter $\epsilon$ ?" In order to address this question, we have performed a series of LS-THC-DF-MP2 calculations on all-trans retinal using the cc-pVDZ basis set $\stackrel{24}{ }$ and corresponding cc-pVDZ-RI auxiliary basis set. $\stackrel{25}{ }$ A large parent grid with 49527 total points (1011 points/atom, $n_{P^{\prime}} / n_{D F}=31.6$ where $n_{D F}$ is the number of auxiliary functions) was used (see SI for details). In fact, this grid has $n_{P^{\prime}}>n_{v} n_{o}$ which, in theory, is enough to exactly fit the $(a i \mid b j)$ integrals. An experimental implementation of LS-THC-DF-MP2 in a development version of the CFOUR program package ${ }^{26}$ was used, and the DF-MP2 results were calculated by reconstruction of the $(a i \mid b j)$ integrals from the transformed DF integrals follow by a conventional MP2 calculation.

The error in the frozen-core LS-THC-DF-MP2 energy compared to canonical DF-MP2 22 is illustrated in figure 1. From these results we can see that the total error decreases approximately linearly with the square of the cutoff $\epsilon$. This is understandable since a cutoff of $\epsilon$ will disregard a residual component of $S$ with diagonal elements at most $\epsilon^{2}\|S\|_{\max }$. The magnitude of the residual diagonal elements is also closely related to the magnitude of the eigenvalues of the rejected portion, such that $\epsilon^{2}$ can be considered as roughly equivalent to the cutoff used in the pseudo-inversion approach. Below $\epsilon \approx 10^{-5}$, the accuracy begins to degrade rapidly. This is likely due to numerical stability issues encountered during the Cholesky decomposition or the least-squares fitting. In some applications, the related $L D L^{T}$ decomposition can enhance numerical stability for positive semi-definite problems, as it can capture the small negative diagonal elements that spuriously arise due to round-off error. 
We intend to explore such a decomposition in further work, although the achievable accuracy seems to be entirely sufficient in this case.

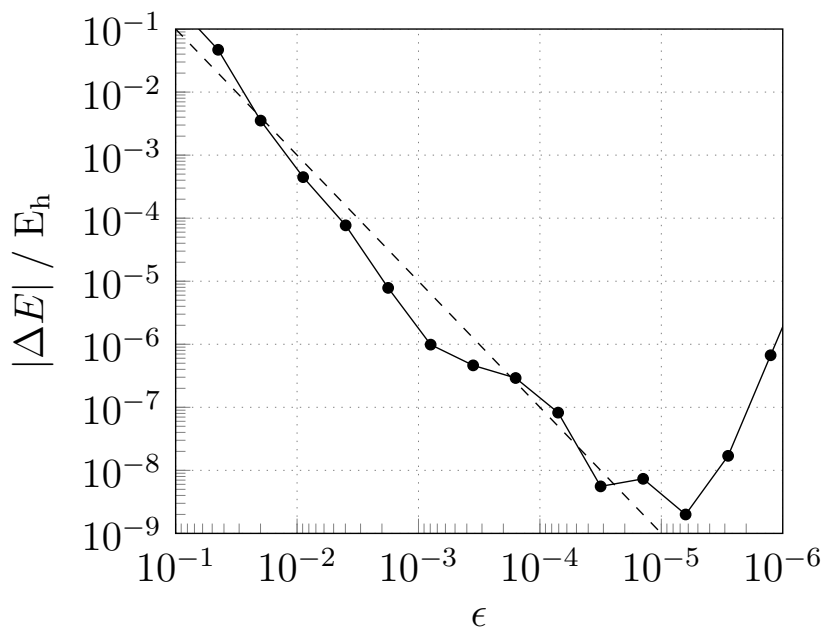

Figure 1: Accuracy of the LS-THC-DF-MP2 approximation compared to canonical DF-MP2 for all-trans retinal as a function of the Cholesky cutoff parameter $\epsilon$. The dashed line shows the linear relation $\Delta E=10 \mathrm{E}_{\mathrm{h}} \times \epsilon^{2}$.

The size of the pruned (ab|, (ai), and $(i j \mid$ grids for different choices of $\epsilon$ are highly linear w.r.t. $\log \epsilon$ below $\epsilon=0.01$. The number of grid points per atom are closely fit by $n_{P}^{(a b \mid} \approx-55 \log \epsilon-21, n_{P}^{(a i \mid} \approx-37 \log \epsilon-39$, and $n_{P}^{(i j \mid} \approx-6 \log \epsilon-6$, which gives $\sim 254,146$, and 24 points/atom at $\epsilon=10^{-5}$ respectively $\left(\sim 8,4.5\right.$, and $\left.0.75 n_{D F}\right)$. This (ai $\mid$ grid is of similar size to the grids used in the original work on LS-THC which were hand-optimized to reduce the error and required grid size. In this case, the tedious hand optimization process is entirely replaced by an automated grid optimization, with tunable error control.

The effect of the size of the starting grid on the pruned (ai $\mid$ grid and the relationship to the orbital basis set were investigated using benzene, coupled with cc-pVXZ basis sets with $\mathrm{X}=\mathrm{D}, \mathrm{T}, \mathrm{Q}, 5$, and 6 and their respective cc-pVXZ-RI auxiliary basis sets. For each basis set, we performed calculations with 17 different parent grids, ranging from relatively small (187 points/atom) to very large (1648 points/atom); $\epsilon=10^{-5}$ was used throughout. For each basis set, increasing the grid size eventually lead to a saturation of the pruned grid, and a plateau in the error w.r.t. canonical DF-MP2. While the largest grid is enough for an 
"exact" decomposition even with cc-pV6Z, the saturated grids reliably prune $\sim 60 \%$ of the parent grid points, leading to between 115 and 615 points/atom. The pruned grid size in units of $n_{D F}$ dropped from $\sim 4.5$ at cc-pVTZ (cc-pVDZ is too close to the exact limit for a reasonable comparison) down to $\sim 3$ at cc-pV6Z. The saturated grids and pruning fraction suggest reasonable sizes for the starting grid in the range $7.5 \leq n_{P^{\prime}} / n_{D F} \leq 11$, although optional starting grid optimization ${ }^{18}$ may also reduce starting grid size.

\section{Conclusions}

We have presented a modification of the LS-THC fitting procedure which leads to automated pruning of the parent grid to grids specific to the $(a b \mid,(a i \mid$, and $(i j \mid$ charge distributions. The extent of pruning is controlled by the cutoff parameter $\epsilon$, the size of the parent grid, and the orbital basis set employed. For a large starting grid, the error is highly linear w.r.t $\epsilon$, and the size of the pruned grids are also linear w.r.t. $\log \epsilon$. As the size of the parent grid increases, the pruned grid quickly reaches a saturation point, with final size (for the (ai $\mid$ distribution) between $3 n_{D F}$ and $4.5 n_{D F}$. This technique shows promise both as a method for in situ generation of optimal grids, and as a tool for facilitating the definition of pre-generated grids for various basis sets and levels of accuracy - in each case the ability to generate distinct grids for different charge distributions is a novel characteristic.

\section{References}

(1) Hohenstein, E. G.; Parrish, R. M.; Martínez, T. J. Tensor Hypercontraction Density Fitting. I. Quartic Scaling Second- and Third-Order Møller-Plesset Perturbation Theory. J. Chem. Phys. 2012, 137, 044103.

(2) Friesner, R. A. Solution of Self-Consistent Field Electronic Structure Equations by a Pseudospectral Method. Chemical Physics Letters 1985, 116, 39-43. 
(3) Ringnalda, M. N.; Belhadj, M.; Friesner, R. A. Pseudospectral Hartree-Fock Theory: Applications and Algorithmic Improvements. J. Chem. Phys. 1990, 93, 3397-3407.

(4) Martinez, T. J.; Mehta, A.; Carter, E. A. Pseudospectral Full Configuration Interaction. J. Chem. Phys. 1992, 97, 1876-1880.

(5) Martinez, T. J.; Carter, E. A. Pseudospectral Mo/Ller-Plesset Perturbation Theory through Third Order. J. Chem. Phys. 1994, 100, 3631-3638.

(6) Martinez, T. J.; Carter, E. A. Pseudospectral Multireference Single and Double Excitation Configuration Interaction. J. Chem. Phys. 1995, 102, 7564-7572.

(7) Neese, F.; Wennmohs, F.; Hansen, A.; Becker, U. Efficient, Approximate and Parallel Hartree-Fock and Hybrid DFT Calculations. A 'Chain-of-Spheres' Algorithm for the Hartree-Fock Exchange. Chemical Physics 2009, 356, 98-109.

(8) Parrish, R. M.; Hohenstein, E. G.; Martínez, T. J.; Sherrill, C. D. Tensor Hypercontraction. II. Least-Squares Renormalization. J. Chem. Phys. 2012, 137, 224106.

(9) Hohenstein, E. G.; Kokkila, S. I. L.; Parrish, R. M.; Martínez, T. J. Quartic Scaling Second-Order Approximate Coupled Cluster Singles and Doubles via Tensor Hypercontraction: THC-CC2. J. Chem. Phys. 2013, 138, 124111.

(10) Hohenstein, E. G.; Kokkila, S. I. L.; Parrish, R. M.; Martínez, T. J. Tensor Hypercontraction Equation-of-Motion Second-Order Approximate Coupled Cluster: Electronic Excitation Energies in O(N4) Time. J. Phys. Chem. B 2013, 117, 12972-12978.

(11) Parrish, R. M.; Sherrill, C. D.; Hohenstein, E. G.; Kokkila, S. I. L.; Martínez, T. J. Communication: Acceleration of Coupled Cluster Singles and Doubles via OrbitalWeighted Least-Squares Tensor Hypercontraction. J. Chem. Phys. 2014, 140, 181102.

(12) Hohenstein, E. G.; Parrish, R. M.; Sherrill, C. D.; Martínez, T. J. Communication: 
Tensor Hypercontraction. III. Least-Squares Tensor Hypercontraction for the Determination of Correlated Wavefunctions. J. Chem. Phys. 2012, 13\%, 221101.

(13) Schutski, R.; Zhao, J.; Henderson, T. M.; Scuseria, G. E. Tensor-Structured Coupled Cluster Theory. J. Chem. Phys. 2017, 147, 184113.

(14) Song, C.; Martínez, T. J. Reduced Scaling CASPT2 Using Supporting Subspaces and Tensor Hyper-Contraction. J. Chem. Phys. 2018, 149, 044108.

(15) Shenvi, N.; van Aggelen, H.; Yang, Y.; Yang, W. Tensor Hypercontracted ppRPA: Reducing the Cost of the Particle-Particle Random Phase Approximation from O(r 6) to O(r 4). J. Chem. Phys. 2014, 141, 024119.

(16) Shenvi, N.; van Aggelen, H.; Yang, Y.; Yang, W.; Schwerdtfeger, C.; Mazziotti, D. The Tensor Hypercontracted Parametric Reduced Density Matrix Algorithm: CoupledCluster Accuracy with O(R4) Scaling. J. Chem. Phys. 2013, 139, 054110.

(17) Parrish, R. M.; Hohenstein, E. G.; Martínez, T. J.; Sherrill, C. D. Discrete Variable Representation in Electronic Structure Theory: Quadrature Grids for Least-Squares Tensor Hypercontraction. J. Chem. Phys. 2013, 138, 194107.

(18) Kokkila Schumacher, S. I. L.; Hohenstein, E. G.; Parrish, R. M.; Wang, L.-P.; Martínez, T. J. Tensor Hypercontraction Second-Order Møller-Plesset Perturbation Theory: Grid Optimization and Reaction Energies. J. Chem. Theory Comput. 2015, 11, 3042-3052.

(19) Lu, J.; Ying, L. Compression of the Electron Repulsion Integral Tensor in Tensor Hypercontraction Format with Cubic Scaling Cost. Journal of Computational Physics 2015, 302, 329-335.

(20) Lu, J.; Thicke, K. Cubic Scaling Algorithms for RPA Correlation Using Interpolative Separable Density Fitting. Journal of Computational Physics 2017, 351, 187-202. 
(21) Feyereisen, M.; Fitzgerald, G.; Komornicki, A. Use of Approximate Integrals in Ab Initio Theory. An Application in MP2 Energy Calculations. Chemical Physics Letters 1993, 208, 359-363.

(22) Kendall, R. A.; Früchtl, H. A. The Impact of the Resolution of the Identity Approximate Integral Method on Modern Ab Initio Algorithm Development. Theor Chem Acta 1997, $97,158-163$.

(23) Harbrecht, H.; Peters, M.; Schneider, R. On the Low-Rank Approximation by the Pivoted Cholesky Decomposition. Applied Numerical Mathematics 2012, 62, 428-440.

(24) Dunning, T. H. Gaussian Basis Sets for Use in Correlated Molecular Calculations. I. The Atoms Boron through Neon and Hydrogen. J. Chem. Phys. 1989, 90, 1007-1023.

(25) Weigend, F.; Köhn, A.; Hättig, C. Efficient Use of the Correlation Consistent Basis Sets in Resolution of the Identity MP2 Calculations. J. Chem. Phys. 2002, 116, 3175-3183.

(26) Stanton, J. F.; Gauss, J.; Cheng, L.; Harding, M. E.; Matthews, D. A.; Szalay, P. G.; Auer, A. A.; Bartlett, R. J.; Benedikt, U.; Berger, C.; Bernholdt, D. E.; Bomble, Y. J.; Christiansen, O.; Engel, F.; Faber, R.; Heckert, M.; Heun, O.; Hilgenberg, M.; Huber, C.; Jagau, T.-C.; Jonsson, D.; Juse \lius, J.; Kirsch, T.; Klein, K.; Lauderdale, W. J.; Lipparini, F.; Metzroth, T.; Mück, L. A.; O’Neill, D. P.; Price, D. R.; Prochnow, E.; Puzzarini, C.; Ruud, K.; Schiffmann, F.; Schwalbach, W.; Simmons, C.; Stopkowicz, S.; Tajti, A.; Vázquez, J.; Wang, F.; Watts, J. D. CFOUR, Coupled-Cluster Techniques for Computational Chemistry, a Quantum-Chemical Program Package with the Integral Packages MOLECULE (J. Almlöf and P. R. Taylor), PROPS (P. R. Taylor), ABACUS (T. Helgaker, H. J. Jensen, P. Jørgensen and J. Olsen), and ECP Routines by A. V. Mitin and C. van Wüllen; www.cfour.de. 